\title{
Cell Cycle Initiation and Bud Emergence in Saccharomyces cerevisiae
}

\author{
By A. LÖRINCZ† AND B. L. A. CARTER*‡ \\ Genetics Department, Trinity College, Dublin, Ireland
}

(Received 27 October 1982)

\begin{abstract}
The coordination of growth with cell division in Saccharomyces cerevisiae has been examined by investigating the order and interactions of three early events in the cell cycle. 'Start', which is thought to be the central control point from which alternative developmental pathways diverge (Hartwell, 1974), preceded bud emergence by about $15 \mathrm{~min}$ regardless of cell growth rate. Cells growing in rich media showed the greatest increase in volume between 'start' and bud emergence. A nutrient-modulated cell size control event (Lorincz \& Carter, 1979) was executed coincident with, or after, 'start'. Therefore, this latter step appears to be the primary point for cell size control in the cell cycle.
\end{abstract}

\section{INTRODUCTION}

In Saccharomyces cerevisiae cell growth and proliferation are coordinated with alternative developmental pathways at an event termed 'start' (Hartwell, 1974). Cells completing this event are not morphologically distinguishable, although bud emergence normally occurs shortly after 'start'. The role of cell size in 'start' control has usually been assessed by investigating size at bud emergence because this is such a useful marker for cell cycle initiation. Cells attain a critical size before the initiation of DNA synthesis and bud emergence (Johnston et al., 1977). This size is nutrient modulated and positively correlated to growth rate (Johnston et al., 1979; Lorincz \& Carter, 1979). The size control operates just prior to bud emergence and cells not already committed to bud production can rapidly change their critical size requirement in response to alterations in the nutritional environment (Lorincz \& Carter, 1979).

We have investigated the temporal and size relationships between three events in the $S$. cerevisiae cell cycle: 'start', commitment to bud initiation, and bud emergence. The time interval between 'start' and bud emergence was of constant duration at all generation times and there was only a small increase in cell size between the two events as compared with overall cell growth. Our experiments placed the nutrient-modulated control governing cell size at bud emergence, coincident with, or subsequent to, the 'start' event thereby providing further evidence that 'start' is the principal event responsible for cell size control in $S$. cerevisiae.

\section{METHODS}

Cell culture conditions. All experiments were done with the haploid strain of Saccharomyces cerevisiae X2180-1 A (MATa gal2, mal, SUC2, CUPl) which was obtained from the Yeast Genetics Stock Center, Berkeley, Calif., U.S.A. The experiments were performed in Erlenmeyer flasks, with exponentially growing cells in the range $2-8$ $\times 10^{6}$ cells $\mathrm{ml}^{-1}$, incubated at $24^{\circ} \mathrm{C}$ in a shaking (about 250 r.p.m.) water bath. The culture media used were YEPD, YEPG and ethanol medium, which have been described previously (Jagadish \& Carter, 1977). $\alpha$-Factor was a generous gift from J. Piggott.

Synchronous cultures. Cell cultures (2 1) were concentrated by centrifugation to about $50 \mathrm{ml}$ : The cell population was then separated according to size (and stage in the cycle) by centrifuging the cells through a $10-40 \%(\mathrm{w} / \mathrm{v})$ gradient of sorbitol made with culture medium in a zonal rotor (Sebastian et al., 1971). Fractions containing the

$\dagger$ Present address: Department of Biological Sciences, University of California at Santa Barbara, Santa Barbara, California 93106, U.S.A.

$\ddagger$ Present address: G. D. Searle and Co. Ltd, High Wycombe, Bucks, U.K. 
smallest cells were isolated, centrifuged, washed with $20 \mathrm{ml}$ fresh medium and inoculated into fresh medium. Starting cultures consisted entirely of unbudded cells and fluorescent microscopy of samples stained with calcofluor (American Cyanamid) revealed that greater than $99 \%$ of these cells were abscissed buds because they possessed no bud scars (Beran, 1968).

Estimation of the percentage of budded cells. Cells were diluted with fixing solution $[1 \%(\mathrm{v} / \mathrm{v})$ formaldehyde, $0.9 \%$

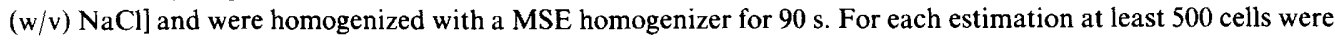
scored with a light microscope at $800 \times$ magnification.

Cell volume measurement. (a) Photomicroscopy. Samples were centrifuged and resuspended in about $1 \mathrm{ml}$ of calcofluor in water $\left(2 \mathrm{mg} \mathrm{ml}^{-1}\right)$. Fluorescing cells were photographed with a Zeiss UV microscope (fitted with UG1 and BG38 primary filters and a K30 secondary filter) using Kodak Tri-X-Pan film. The images of the cells were projected on to a screen and their dimensions measured. Cell volumes were calculated using the equation

$$
v=\frac{4}{3}\left(\frac{b m}{2}\right)^{2} \frac{a m}{2}
$$

where $b$ is the length of the minor axis ( $\mathrm{mm}), a$ is the length of the major axis $(\mathrm{mm})$ and $m$ is the magnification conversion factor $\left(\mu \mathrm{m} \mathrm{mm}^{-1}\right)$ calculated by using a calibration graticule photographed at the same magnification as the cells (Graticules Ltd, Kent, U.K.). Cell population volume distributions were found to be normally distributed. Consequently, the volume $v$ at any desired percentile could be calculated using the equation

$$
v=z \sigma+\mu
$$

where $\mu$ is the mean volume, $\sigma$ the standard deviation and $z$ the standard normal deviate; this value is obtained from statistical tables which give $z$ values for any desired percentage (e.g. percentage of budded cells).

(b) Coulter Channelyzer. Cell samples were diluted in Isoton II electrolyte and homogenized. The samples were analysed with a Coulter Channelyzer (Coulter Electronics, Harpenden, Herts, U.K.) using a $70 \mu \mathrm{m}$ diameter aperture tube. The Coulter Channelyzer was calibrated to $\mu \mathrm{m}^{3}$ using synchronous cell populations whose volumes had been calculated by photomicroscopy. A simple summing procedure was employed to estimate the volume at which the distribution was divided into any desired set of percentages. The percentage of cells in each sequentially increasing volume range were summed until the desired value was obtained; the corresponding final volume was noted.

\section{RESULTS}

Temporal and size relationship between the $\alpha$-factor step and bud emergence

In a typical experiment small daughter cells in $\mathrm{G} 1$ phase were isolated and inoculated into fresh medium to give a synchronous culture. The appearance of budded cells was monitored by microscopy. In one such experiment the culture was divided into two samples when the percentage of budded cells had reached $39 \%$. $\alpha$-Factor was added to one sample to a final

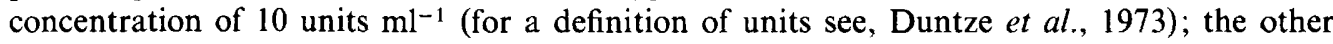
portion was left undisturbed and used as a control. The percentage of budded cells in the $\alpha$-factor treated culture reached a plateau value of $58 \%$ after $50 \mathrm{~min}$ (Fig. 1). The difference between this plateau value and the value at the time of $\alpha$-factor addition represents the number of cells in the population which at the time of challenge were in the interval between the $\alpha$-factor arrest point (the $\alpha$-factor step) and bud emergence. The time taken for the percentage of budded cells in the control culture to increase from $39 \%$ to $58 \%$ defines the time interval between these two events and was estimated as $13 \mathrm{~min}$.

To obtain a more accurate estimate of the above time interval, $\alpha$-factor was added to samples removed at intervals from a synchronously budding cell population. The percentage of budded cells in the treated cultures was scored $60 \mathrm{~min}$ after addition of $\alpha$-factor since at this time a transient plateau was reached (see Fig. 1). The results obtained for a synchronous cell population growing in YEPD medium are shown in Fig. 2. The stage of development of the parent culture at the time of shift to $\alpha$-factor had no effect on the duration of the time interval between the $\alpha$ factor step (start) and bud emergence and was an average of $13.5 \mathrm{~min}$.

Similar shifts were done for cells growing in media supporting slower generation times. In all cases bud emergence followed soon after the $\alpha$-factor step and the time interval between the events appeared to be independent of the generation time (Table 1).

Cell volume at bud emergence can be determined directly by photomicroscopy (Johnston $e t$ al., 1979; Lorincz \& Carter, 1979). However, this procedure is not possible for assessing cell size 


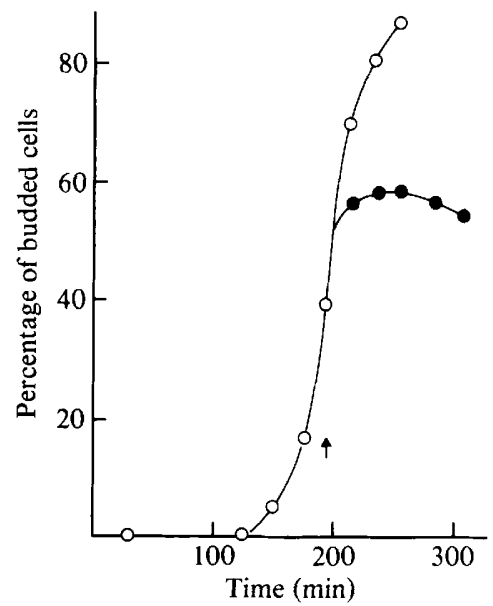

Fig. 1

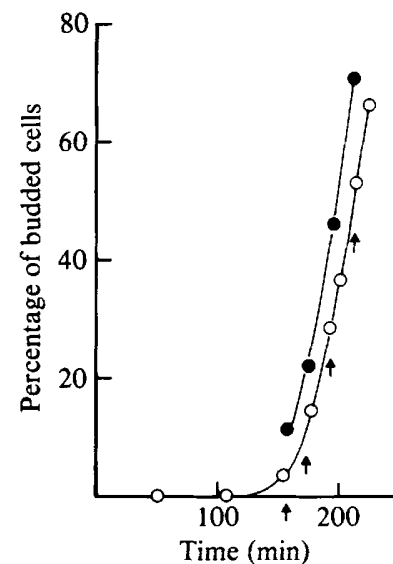

Fig. 2

Fig. 1. Challenge with $\alpha$-factor, of a synchronously budding population of cells in YEPD medium. Percentage of budded cells: control culture $(O)$, sample challenged with $\alpha$-factor $(O)$. The time of addition of $\alpha$-factor is indicated by the arrow.

Fig. 2. Addition of $\alpha$-factor to samples removed from a synchronously budding cell population in YEPD medium. Percentage of budded cells : parent culture $(O)$, cultures which received $\alpha$-factor $(O)$. The percentage of budded cells in these latter cell populations was determined 60 min after the shift to $\alpha$-factor, at which time the percentage of budded cells had reached a plateau value. The arrows indicate the time of addition of $\alpha$-factor. The increase in the percentage of budded cells after $\alpha$-factor addition represents the percentage of cells in the population which were in the interval between the $\alpha$-factor step and bud emergence at the time of challenge.

Table 1. Relationship between the $\alpha$-factor step and bud emergence in cells growing at different generation times

Growth medium ... YEPD*

Generation time (min)

Time-interval between the $\alpha$-factor step and bud emergence (min)

Median volume of cells immediately after isolation $\left(\mu \mathrm{m}^{3}\right)$

Volume at the $\alpha$-factor step $\left(\mu \mathrm{m}^{3}\right)$

Volume at bud emergence $\left(\mu \mathrm{m}^{3}\right)$
YEPG†

275

$13.7 \pm 0.31$ S.E.

$14 \cdot 4$

$37 \cdot 6$

$40 \cdot 3$
Ethanol*

$15.9 \pm 0.83$ S.E.

$11 \cdot 8$

$27 \cdot 3$

28

* These data are the average values for four different experiments.

$\dagger$ These data are the average values for two different experiments.

at the $\alpha$-factor step since these cells do not exhibit any distinguishable morphological features. Because the volume profiles of cell populations fitted a normal distribution having a mean and a standard deviation, the volume of cells at the $\alpha$-factor step could be estimated using equation 2 (see Methods). In the experiment shown in Fig. 1 the cell population when $39 \%$ of the cells were budded had a mean volume and standard deviation of $39.8 \pm 6.3 \mu \mathrm{m}^{3}$. At this time $58 \%$ of the cells had volumes greater than $38.5 \mu \mathrm{m}^{3}$ which was therefore taken as the volume of the cells at the $\alpha$-factor step. Similarly, $41.6 \mu \mathrm{m}^{3}$ was calculated as the volume of cells at bud emergence.

In other experiments an alternative procedure (which gave similar results) involving a Coulter Channelyzer, was employed. The population distribution obtained with the Channelyzer confirmed that synchronous populations exhibited a normal volume distribution until cell division. As with the previous procedure the volume analysis was performed with the cell population at the time of challenge with $\alpha$-factor. The percentage of cells which had completed the event of interest was noted and by assuming that these were the largest cells, the volume at that event was estimated. 


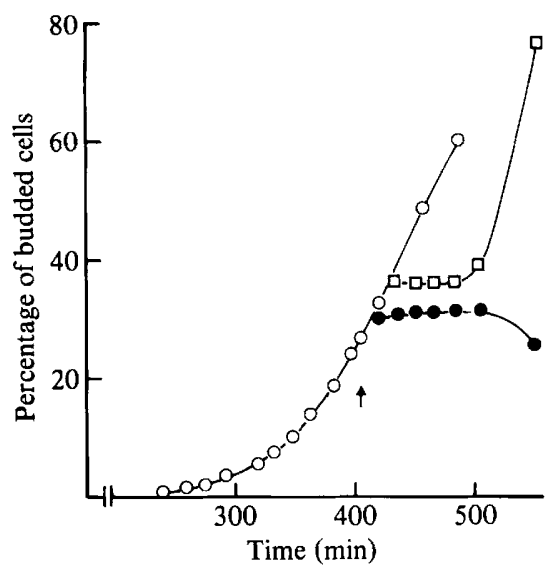

Fig. 3. Transfer of samples of a synchronously budding cell population in ethanol medium, to YEPD medium with (O) or without $(\square) \alpha$-factor. The parent culture in ethanol medium is shown by (O). The arrow shows the time of the transfers. The increase in the percentage of budded cells after the shift to YEPD addition represents the percentage of cells in the population which were between the size commitment step and bud emergence.

The data for cell cultures growing at different generation times is presented in Table 1. As expected the cell volume at bud emergence was a little larger than at the $\alpha$-factor step; however, this increase was slightly greater for cells growing at faster generation times.

\section{Ordering of bud initiation with respect to the $\alpha$-factor step}

Commitment to bud initiation is defined as the point in the cell cycle at which cells become committed to producing a bud at a particular parental volume even if the nutritional environment is changed to one which supports a larger cell size at bud emergence (Lorincz \& Carter, 1979). Therefore, size control in $S$. cerevisiae must occur at, or prior to, this event. Since the conditions for $\alpha$-factor arrest and nutrient modulation of parental cell size can be independently imposed, it was possible to investigate the relationship between the $\alpha$-factor step and the event sensitive to nutrient modulation (i.e. commitment to bud initiation).

A synchronous culture of small daughter cells in ethanol medium was prepared and at intervals this culture was monitored for the appearance of buds. When the percentage of budded cells reached $27 \%$, two samples were removed from the culture, the remainder of which was left as a control. The samples were filtered and the cells from one were resuspended in fresh prewarmed YEPD medium; the cells from the other were resuspended in fresh prewarmed YEPD medium containing 4 units of $\alpha$-factor $\mathrm{ml}^{-1}$. The data are shown in Fig. 3. The percentage of budded cells in the culture shifted to YEPD medium increased from $27 \%$ to a plateau value of $36 \%$. After a period of $90 \mathrm{~min}$ post-shift the percentage of budded cells in this culture began to increase again at a considerably faster rate than in the control culture. The percentage of budded cells in the shift to YEPD medium containing $\alpha$-factor increased from $27 \%$ to a plateau value of $31 \%$ and started to decrease at about $100 \mathrm{~min}$ post-shift. These results are interpreted as follows: the sample which received YEPD medium only, exhibited a response typical of a shift to a richer nutritional medium (Lorincz \& Carter, 1979). The cells which were already committed to producing buds at a parental cell size characteristic of ethanol medium (about 30\% smaller than in YEPD medium) did so, hence the increase in budded cells to a plateau value of $36 \%$. However, the cells which had not reached this commitment event reset their size control such that a larger volume was required for commitment to bud initiation. The plateau represents the time period during which these cells grew to the new larger size after which bud emergence occurred at a faster rate typical of that in YEPD medium. In the culture shifted to YEPD medium containing $\alpha$-factor, the cells prior to the $\alpha$-factor step arrested and remained unbudded. A number of unbudded cells which had already completed the $\alpha$-factor step 


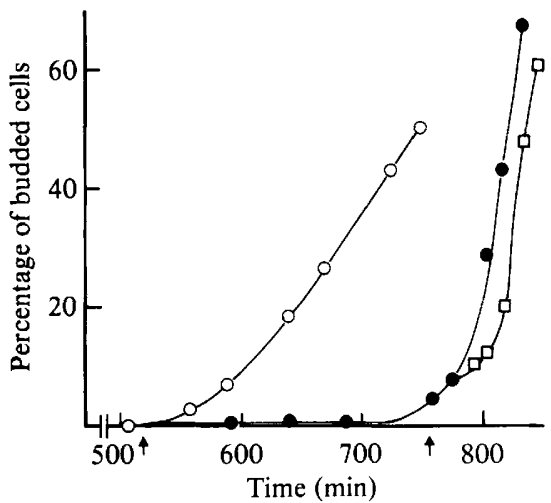

Fig. 4

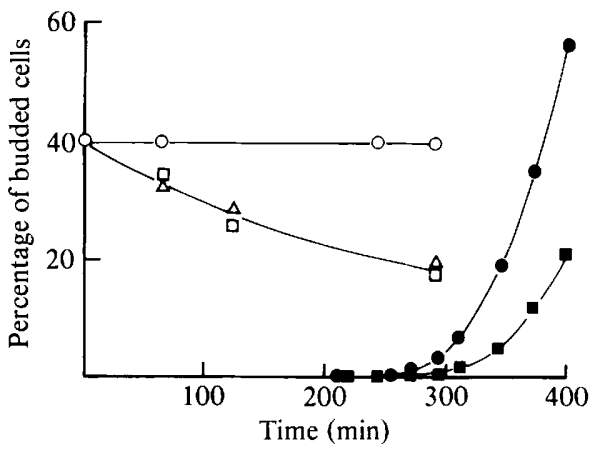

Fig. 5

Fig. 4. The ability of cells arrested by $\alpha$-factor in ethanol medium to respond to nutrient enrichment after spontaneous recovery from arrest. $\alpha$-Factor was added to a culture just prior to synchronous bud protection in ethanol medium (shown by the arrow on the left). When the cells began to recover from arrest a sample was enriched with YEPD medium (shown by the arrow on the right). Percentage of budded cells: in ethanol medium (O), in the culture recovering from $\alpha$-factor arrest in ethanol medium $(\odot)$ and in YEPD enriched medium ( $\square$ ).

Fig. 5. An investigation of the potency of and recovery from $\alpha$-factor induced cell cycle arrest in ethanol and YEPD medium. Samples from an exponentially growing culture of cells in ethanol medium were transferred to ethanol or YEPD medium containing $\alpha$-factor. The control and $\alpha$-factor challenged cultures were monitored at intervals for the percentage of budded cells: control culture $(O)$, culture in ethanol medium containing $\alpha$-factor $(\square)$, culture transferred to YEPD medium containing $\alpha$-factor $(\triangle)$. The percentage of newly formed budded cells after recovery from mating pheromone arrest in YEPD medium (O) and in ethanol medium $(\boldsymbol{\square})$ is shown.

produced buds before a plateau was reached. The higher plateau value for percentage of budded cells in the sample which received YEPD medium alone indicates that in the pre-shift culture there was a greater percentage of cells in the interval between the point of commitment to bud initiation and bud emergence than between the $\alpha$-factor step and bud emergence. These data suggest that commitment to bud initiation occurs before the $\alpha$-factor step by about $11 \mathrm{~min}$ (using the method described earlier to estimate the time intervals). It is possible, however, that the response of cells to the nutritional shift is slower than the response to $\alpha$-factor. This would have the effect of increasing the apparent number of cells between commitment to bud initiation and bud emergence, and would result in an erroneous estimate of the time interval between these two events.

We investigated this latter possibility and our rationale for the experiment was as follows: if commitment to bud initiation precedes the $\alpha$-factor step then cells arrested by $\alpha$-factor in a medium supporting a small parental cell size at bud emergence should be unable to respond to a richer medium after release from the arrest. In contrast if the order of events is the reverse, or if they are coincident, then these arrested cells should be responsive to the richer medium and if enriched with YEPD medium should produce buds at a larger size. For the experiment a synchronous population of cells in ethanol medium was prepared. The culture was examined periodically for the appearance of budded cells. When the culture had just started to produce buds ( $0-1 \%$ buds) a low concentration of $\alpha$-factor $\left(2\right.$ units $\left.\mathrm{ml}^{-1}\right)$ was added to a sample taken from the parent culture. This concentration of mating pheromone allows spontaneous recovery from $\alpha$-factor arrest 3-4 h later. Both the parent culture and the $\alpha$-factor treated culture were monitored for percentage of budded cells (Fig. 4). When cells began to spontaneously recover from $\alpha$-factor arrest (recognized by the appearance of small buds) the treated culture was divided into two samples. The first sample was diluted $1: 1$ with fresh YEPD medium, this mixed medium supports the same generation time and cell size at bud emergence as YEPD medium. The second sample was left as a control to determine the kinetics of recovery from $\alpha$-factor arrest in ethanol medium. The addition of YEPD medium delayed bud emergence by about $30 \mathrm{~min}$ 
compared to the control culture in $\alpha$-factor treated ethanol medium. This implies that the cells recovering from $\alpha$-factor arrest imposed in ethanol medium were smaller than the size required for bud initiation in the enriched medium and that the delay in bud emergence represents the time interval during which the cells grow to the size required for bud initiation in the enriched medium. This places the nutrient-sensitive size regulated step at, or subsequent to, the $\alpha$-factor step in the $S$. cerevisiae cell cycle.

It was possible that the observed delay in bud production after the addition of YEPD medium was due to a short lag period of no growth caused by the shift, or alternatively, to an increased sensitivity to $\alpha$-factor in YEPD medium as compared to ethanol medium. The first possibility was excluded by a volume analysis of the cells during the course of the experiment described. The cells recovering from $\alpha$-factor arrest imposed in ethanol medium had grown significantly after the enrichment and produced buds at the size characteristic of cells in YEPD medium (data not shown).

To examine the relative potency of $\alpha$-factor arrest in ethanol versus YEPD medium, an experiment was performed with asynchronous cells. An exponentially growing culture in ethanol medium was divided into three samples. The first was left undisturbed as a control culture. The second received $\alpha$-factor to a concentration of 5 units $\mathrm{ml}^{-1}$ and the third was filtered and resuspended in YEPD medium containing 5 units $\alpha$-factor $\mathrm{ml}^{-1}$. The number of cells per $\mathrm{ml}$ for all three cultures was the same. The percentage of budded cells in the control culture remained at approximately $40 \%$ during this experiment, whereas in the two cultures treated with $\alpha$-factor it decreased at approximately the same rate (Fig. 5). After a period of growth (about $200 \mathrm{~min}$ ), the cell morphologies in $\alpha$-factor treated cultures had changed markedly, almost all the cells had become shmoos (irregularly shaped cells indicative of $\alpha$-factor arrest). The budded cells still remaining at this time appeared as two attached shmoos. Recovery from $\alpha-$ factor induced cell cycle arrest was monitored by the appearance on cells of small protuberances which rapidly enlarged into buds. In the YEPD culture recovery was first noticed at about 230 min after the addition of $\alpha$-factor, whereas in the ethanol culture it began at approximately $270 \mathrm{~min}$. The production of new buds was more rapid in YEPD than in ethanol medium due to the faster generation time supported by the former. This experiment demonstrates that $\alpha$-factor elicits a slightly longer arrest period in ethanol medium.

It appears that cell size is stringently regulated at the $\alpha$-factor step. The erroneous order obtained from the experiment shown in Fig. 3 was due to a slower response of the cells to nutritional enrichment than to the mating pheromone. If the $\alpha$-factor step occurs prior to the point of commitment to bud initiation it still follows that it is size regulated because this former step occurs shortly before bud emergence in cells growing at both faster and slower generation times; these growth conditions support different characteristic cells sizes at bud emergence.

\section{DISCUSSION}

Two events that occur just prior to bud emergence in the cell cycle of $S$. cerevisiae are 'start' (Hartwell, 1974) and a nutrient modulated size control step (Johnston et al., 1979; Lorincz \& Carter, 1979). Traversal of 'start' is characterized by the simultaneous completion of the $c d c 28$ event and progression to $\alpha$-factor insensitivity (the $\alpha$-factor step; Hereford \& Hartwell, 1974). Completion of the nutrient-modulated size control step is marked by a commitment of cells to bud production at the parental size characteristic of the medium in which the event was executed.

We have explored the temporal relationship between 'start', commitment to bud initiation, and bud emergence. Our results revealed that 'start' precedes bud emergence by about 15 min for all the generation times examined. Hartwell \& Unger (1977) examined the length of this interval in cells growing at four doubling times within the range 198-714 min using different concentrations of the protein synthesis inhibitor cycloheximide to vary generation time on solid agar media. Time-lapse photomicroscopy was used to show that the time interval between 'start' and bud emergence varied between 20-30 min and there was no apparent trend with generation 
time. The discrepancy between the average time interval estimated in the above study (average $24 \mathrm{~min}$ ) and that described here $(15 \mathrm{~min})$ can probably be attributed to differences in experimental procedures.

Simultaneous transfer of cells from ethanol medium to YEPD medium with or without $\alpha$ factor suggested that commitment to bud initiation occurred prior to 'start' (the $\alpha$-factor step). Subsequent experiments revealed, however, that the $\alpha$-factor step is in fact coincident with, or prior to, commitment to bud initiation. Our initially incorrect ordering of these events occurred because cells responded faster to $\alpha$-factor than to nutritional stimulus. It was not possible to distinguish between the two remaining alternatives for the order of the $\alpha$-factor step with respect to commitment to bud initiation as the reciprocal shift protocol (Jarvick \& Botstein, 1973) could not be used. Nevertheless, 'start' must be a nutrient-modulated size control point as cells arrested at this event can respond to a nutritional shift by resetting their size requirement for bud emergence.

Cells recover spontaneously from $\alpha$-factor arrest in ethanol medium only slightly later than in YEPD medium even though there is close to a threefold difference in generation time in these media. Thus, there appears to be a rate limiting event in the $\alpha$-factor recovery process (requiring endopeptidase action; Ciejek \& Thorner, 1979) which is independent of cell mass and generation time.

This research was supported by a grant from the Irish Cancer Society.

\section{REFERENCES}

BERAN, K. (1968). Budding of yeast cells, their scars and ageing. Advances in Microbial Physiology 2, 143-171.

Ciejek, E. \& Thorner, J. (1979). Recovery of $S$. cerevisiae $a$ cells from $\mathrm{Gl}$ arrest by $\alpha$-factor requires endopeptidase action. Cell 18, 623-635.

Duntze, W., Stotzler, D., Bucking-Throm, E. \& KalbitZer, S. (1973). Purification and partial characterization of $\alpha$-factor, a mating type specific inhibitor of cell reproduction from Saccharomyces cerevisiae. European Journal of Biochemistry 35, 357365.

HarTwell, L. H. (1974). Saccharomyces cerevisiae cell cycle. Bacteriological Reviews 38, 164-198.

HarTwell, L. H. \& UNGER, M. W. (1977). Unequal division in Saccharomyces cerevisiae and its implications for the control of cell division. Journal of Cell Biology 75, 422-435.

Hereford, L. M. \& Hartwell, L. H. (1974). Sequential gene function in the initiation of Saccharomyces cerevisiae DNA synthesis. Journal of Molecular Biology 84, 445-461.

J AGADISH, M. N. \& CARTER, B. L. A. (1977). Genetic control of cell division in yeast cultured at different growth rates. Nature, London 269, 145-147.

JARVICK, J. \& BotSTEIN, D. (1973). A genetic method for determining the order of events in a biological pathway. Proceedings of the National Academy of Sciences of the United States of America 70, 20462050.

Johnston, G. C., Pringle, J. R. \& Hartwell, L. H. (1977). Coordination of growth with cell division in the yeast Saccharomyces cerevisiae. Experimental Cell Research 105, 79-98.

Johnston, G. C., Ehrhardt, C. W., Lorincz, A. \& CARTER, B. L. A. (1979). Regulation of cell size in the yeast Saccharomyces cerevisiae. Journal of Bacteriology 137, 1-5.

LORINCZ, A. \& CARTER, B. L. A. (1979). Control of cell size at bud initiation in Saccharomyces cerevisiae. Journal of General Microbiology 113, 287-295.

Sebastian, J., Carter, B. L. A. \& Halvorson, H. O. (1971). Use of yeast populations fractionated by zonal centrifugation, to study the cell cycle. Journal of Bacteriology 108, 1045-1050. 